\title{
Measure inegality in the investors' risk aversion and behavioral heterogeneity: method and experimentation
}

\author{
JihèneJebenianiGouider1, MokhtarKouki2 \\ Institute of Higher Business Studies, Carthage University, Carthage Presidence2016 Tunis, Tunisia. \\ Higher Institute of StatisticsandInformation Analysis, Carthage University, Tunisia.
}

\begin{abstract}
ASTRACT
This article studies the inequalities in measurements of the risk aversion in the context of the financial investments in Tunisia. We clarify initially the factors constitutive of the risk aversion. The studied actors are individual decision makers. The tackled questions are the risk attitude (including the risks known as extremes), its perception, its evaluation, the decision-making in risky universe. The empirical data were collected through experimental sessions carried out in Tunisia. We propose a framework of analysis for the study of the investors' preferences based on an operational econometric modeling. The estimated models are the ordered probit and the ordered probit with random effects. The model with random effects has the advantage of making it possible to test the heterogeneity of the individuals and to measure the inequality in risk aversion of the investors, and this, by studying the components between and within-individual of the variance of the risk aversion.
\end{abstract}

KEYWORDS: risk aversion, behavioral finance, experimental economy, lotteries, ordered probit choice model, random effects orderdprobit choice model.

\section{Academic Discipline And Sub-Disciplines :}

Behavioral finance; Psychology; econometric model; experimental economy

\section{TYPE}

Experimental approach, econometric studies

\section{Council for Innovative Research}

\author{
Peer Review Research Publishing System
}

Journal of Social Science Research

Vol.3, No.2

editor@jssronline.com

www.cirworld.com, www.jssronline.com 


\section{INTRODUCTION}

The study of the financial sector and analysis of the behaviors of the individuals who work, arouse today an interest growing of partly with the recent trends in the capital market (see Eeckhoudt, Gollier and al., 2005; Gollier, 2001). Although the aversion with risk is a key factor, determining the investors' behavior is difficult to rigorously measure the risk aversion in the context of financial investments. Indeed, the risk aversion is expressed according to two dimensions, one economic related to the liquidity or the stability of the income, the other subjective one expressed the psychological aptitude of the investor to be lost (see Broihanne, Merli and Roger, 2004; Step, 2005). One of the principal difficulties in the measurement of aversion to the risk is related to its subjective nature that can be measured only by using the questionnaires trying to capture the determining elements in the investors' behavior.

The majority of the evaluation methods of the risk aversion developed at the present time rest on models, which expressly suppose a linearity between dependent variable and explanatory variables. Such models cannot be adequate since one takes account of subjective nature of risk aversion. In practice, the approach most used by the financial intermediaries is that of the scoring ${ }^{3}$ question. The financial institutions proposeto their customers a small number (from 3 to 6 ) of standard wallets, which are positioned along a one-dimensional scale of risk. An optimal position corresponds to the minimization of the average of the cost for the investors who do not have access to a wallet entirely personalized (see of Palma and Prigent; 2008).

Our analysis exceeds the existing literature on the estimate of risk aversion by proposing an approach that combines at the same time the techniques of experimental economy (of the questions of the lotteries type based on the risky and nonrisky credits and questions related to the attitudes) (see of Palma and Picard 2002, 2005) and the flexibility of discrete choice models (see Anderson, of Palma and Thisse, 1992; McFadden, 2001; Ben-Akiva, McFadden and of Palma 2003). Indeed, there is an immense dissymmetry between analysis of the purely financial risk aversion (cognitive process) and econometric analysis. Our approach is compatible at the same time with the expected utility theory (see Von Neumann and Mogenster, 1947) and non-expected utility theory (see Kahneman and Tversky, 1979; Tversky and Kahneman, 1992). Within the framework of non-expected theory, we use for our problems to cumulated prospects theory (CPT), proposed by Kahneman and Tversky (1992) ${ }^{4}$. In addition, certain researches developed estimate of risk aversion comparable with that used in this work, but which are applicable either within the framework of expected utility theory (see Hartog, Ferrer-i-Carbonell and Jonketand, 2000), or within the framework non-expected utility theory (see Donkers, Melenberg and van Soest; 2000).

However, several work tried to extend the models of existing choices, in a choice model which stresses the fact that the choice is controlled by perceptions. These models treat also heterogeneity through the decision makers like the various attitudes and perceptions and of significant latent constructions due to the perceptions influenced by psychological factors and external constraints, Train and McFadden (2000), Green (2002). Thus, the latent factors provide a richer behavioral representation process of choice and the addition of the tastes of heterogeneity improves the explanatory power of the model. Key contribution is the development of the model of choice, whose results of estimate show that the individuals differ in their risk attitudes. From where, considerable interest to model explicitly heterogeneity.

In this article, we lay down a double objective: we propose another manner of generalizing the canonical model of expected utility as well as the model of non-expected utility. This extension leaves the following observation: if we put the same questionwith an individual, or a series of questions logically related to the same individual, we generally observe a dispersion of answers around an average answer. Typically, the same individual confronted with identical conditions will answer differently to the same questions during different occasions. The classical expected utility theory, or the non-expected utility theory, is not able to give an account of this simple observation (see for example Pfiffelman, 2008). We can argue that individuals learn, or although a certain tiredness settles or that there are effects of context. But, another answer is possible. It consists in regarding these variations of behaviors as errors, present in any collect data. This taking into explicit account of errors, from the start at the theoretical level, seems significant to us. To give an account of these deviations to the behaviors of the economic theory, we propose to introduce second source of heterogeneity into the population. Indeed, there is an unobserved heterogeneity of the tastes in the population: the parameters of the variables are not invariants with the sampled individual. Also homogeneous is the sample, the relations of preferences subjacent with the levels of utility of the individuals cannot be strictly identical.

The originality of this extension is as it applies as well to the expected utility model as with the non-expected utility model.Thus try to extend the methodology of estimate of Palma and Picard (2005), by introducing the heterogeneity of individuals (see Barsky et al., 1997), by having recourse to the panel techniques to estimate how the risk aversion varies at the same time between individuals and, for the same individual, for several situations of choice. More precisely, we proposeto relax the restrictive assumption of the unicity of the behavioral parameters on the whole of investors. Minks to bring an additional lighting on the role of individual heterogeneity in the measurement of the risk aversion.

In order to control this individual heterogeneity, we carry out a survey which measures and quantifies individual reactions to the situations where risk relates to the financial investment. We measure risk aversion of the same individual face to several situations of choice. In risk aversion, there is a part specific to the investor when he carries out his choices while the other part refers to the context of choice (proper to the lotteries series), to see for example Pfiffelman and Roger(2005). In two different contexts, the same individual makes different choices. We pose to the same individual several series of lotteries, in order to recall the risk aversion of the same individual for various levels of risk, different rising and various durations from investments. We try to measure the inequality in investors' risk aversion by applying a panel model, namely the random effects probit ordered model (see Chamberlain, 1984; Hedeker and Gibbons, 1994), which is one of methodologies generally adopted to consider variables dependent, having a natural order in data of panel (see Ben-Akiva and al, 2001;

\footnotetext{
${ }^{3}$ total score is the sum of the scores obtained with each

${ }^{4}$ results on the test of the theory of utility are not matter of this article
} 
Walker and Ben-Akiva, 2002; Ben-Akiva, McFadden and of Palma, 2008; Ben-Akiva and al., 1999). This study aims at testing the heterogeneity of the individuals. Indeed, we can use the models of panel, as soon as there are several observations for same individual, even if they are collected at the sametime (during an experimental session). We consider two dimensions then: the individual $i$ and the series of lottery $k$. Such are the questions, which we try to answer: what does it occur, if we pose to the same individual two series of different lotteries? Does the random effects always improve the explanatory power of the model? In addition, is there heterogeneity between individuals? If so, how is the significance of explanatory variables affected by non-observables heterogeneity individuals?

The second objective of our article consists in measuring the inequality of risk aversion in the context of financial investments by using the models of panel. Indeed, understand the relative importance of the differences between and within individual of risk aversion is crucial. The impact of individual on his choices depends on the characteristics of within and between individual. However, we know little on the relative magnitudes of inequalities will within and between individual in the risk aversion. Consequently, our interest is carried to the components will within and between individual of risk aversion, still very little explored within the framework specific of our research. With this intention, we carry out a variance analysis on random effects ordered probit model. Our analysis consists of a decomposition of the variance of dependent variable on individual risk aversion, in within and between-individual components of the observable and non-observable components. We will try to estimate the components of the variances within and between individual. In order to study the stability of the individuals' risk aversion of a series to another.

This article arises as follows: the first section will be devoted to the presentation of data of experimental economics of our sample followed by descriptive statistics. The econometric model and its proprietieswill be discussed in the second section. The third section present the results of the estimate of the random effects ordered probit ordered model on experimental tunisian sample: we will start by testing the heterogeneity of the individuals in the sample, then we carry out a comparison between results of the estimate of the model with and without random effects, in order to study the improvement made by the random effects on the explanatory power of the model. Lastly, we carry out a variance analysis will within and between-individualin order to measure the individuals' inequality in risk aversion.

\section{DESCRIPTIVE DATA AND STATISTICS}

\subsection{Presentation of the data: experimental protocol}

In the context of the experimental economy, we developed an experimental laboratory, the first in Tunisia, on the evaluation of the individual risk aversion in the context of the financial investments (see Brook and Zank, 2004; Goeree, Holt and Palfrey, 2003; Holt and Laury, 2003; Luce, 2000; Roth, 1988; Holt, 2006). We conducted experiments ${ }^{5}$ in a guided and controlled way which measured and quantified the individual reactions towards situations where the risk was on the financial investment. Respondent were encouraged to provide sincere answers with a remuneration related to their answers. Indeed, the choices of the individuals determine their earnings. The financing of our experimental sessions was assured by the French National Agency of Research (ANR). The experiments were conducted at the Business school of Tunis, in a computer room equipped of broadband Internet so as to get easily connected the site www.RiskToleranceOnLine.com. However, we adapted the questionnaire of the Web site to the Tunisian context (reference currency, adapting the invested amounts in dinars Tunisian diplomas, ...

The experimental sessions were held from February 2012 to April 2012. Given the planned treatments, 30 experimental sessions; each including between 9 and 15 subjects were conducted. The average duration of the experiments was approximately $1 \mathrm{~h} 30 \mathrm{mn}$. The subjects were mainly students of second degree in economic sciences, finance, the teaching and administrative staff of the universities. We collected 386 respondents, during this experimental laboratory. Each participant responded to 10 series of lotteries. During each session, the subjects made choices to ten series of lotteries. In addition, they were informed of the number of sets of lotteries to be filled. The only modifications among the series of the lotteries game were the return laws of the lotteries and the interval where its returns vary. In the first three series (series 1 to 3 ) the return followed the uniform law, whereas series 4 to 6 the return followed a symmetric binary law. These six series included negative return in order to measure the asymmetry around zero. However, in the series 7 and the 8, the outputs had a small probability of large loss, whereas in series 9 and 10, the return had a small Jackpot probability. The figure (1) shows the decision tree of lotteries. The monetary incentives werevaried according to individual choices. Starting with an initial equal amount for all the respondents that are 30 Tunisian dinars, the final remuneration consisted of the amount that was multiplied by the return corresponding to the choice, which was made, on one of the 10 series of lotteries randomly drawn. The choice of the initial monetary values took into account several potentially contradictory constraints. We carefully selected this initial amount by resorting to excavated pilot studies (with about fifteen subjects). To be remunerated, the individual randomly drew a number between 1 and 10 that corresponded to the series determining his gain.

${ }^{5}$ Experiment is a whole of sessions are in controlled environment. 


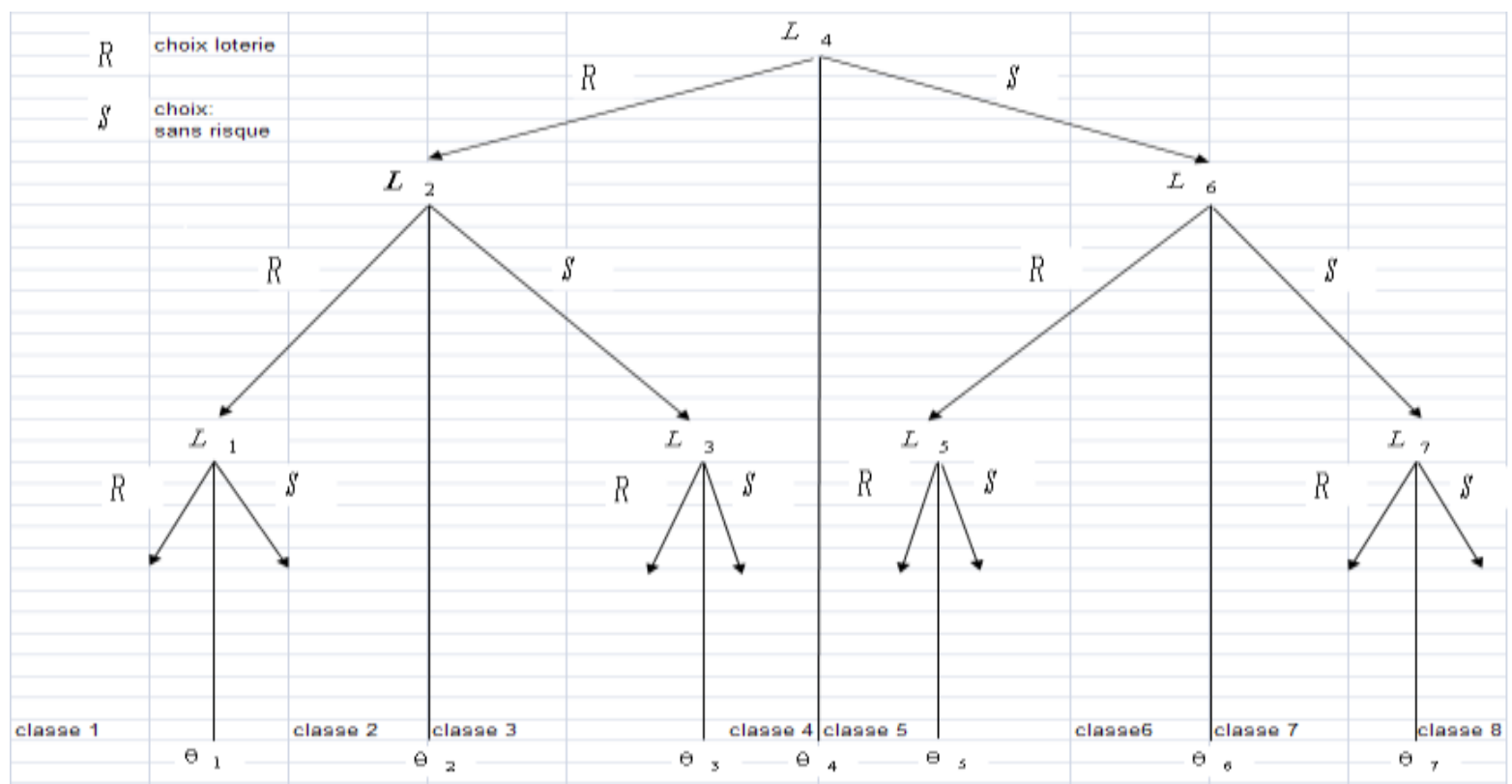

Figure 1. Representation of decision problem.

The incentives are monetary and vary according to choices of the individual. On the basis of an identical initial amount for all the candidates 30 dinars Tunisian, final remuneration is this rising multiplied by the output corresponding to the choice which it made on one of the 10 series of randomly drawn lotteries. The choice of the initial money values took account of several potentially contradictory constraints. We carefully selected this starting amount while resorting to excavated pilot studies (with about fifteen subjects). Obviously, only the individuals who finished the questionnaire will be remunerated. Indeed, after having answered the 10 series of lotteries, a summary table ${ }^{6}$ taking again the questions and answers of the individuals will be posted.

More precisely, to pay the individuals, we make draw the individual randomly a number between 1 and 10 correspondent with the series, which determines its profit:

If the series number drawn randomly, corresponds to a series in the which individual carried out a certain choice, then remuneration is the rising basic one (30 Dinars Tunisian) multiplied by the certain return rate which chooses at the time thisseries.

- $\quad$ But, if the serie number drawn randomly, corresponds to a series in the which individual make a risked choice, whose output follows an equiprobable binary law (series 4, 5 and 6). It is necessary to launch a coin. If it falls on pile, we multiply the negative output by the basic amount and if it falls on face, we multiply the positive rendement by the basic amount.

- In addition, if the series number randomly drawn, whose return is uniform (series 1,2 and 3 ), it is necessary to proceed as follows: let us suppose that the uniform return one between $-5 \%$ and $20 \%$, it is necessary to make it draw a paper in a ballot box containing from paper written $-5 \%,-4.5 \%, \cdot, 19 \%, 19.5 \%, 20 \%$. The return drawn one from the ballot box is multiplied by the basic amount.

- $\quad$ Finally, if the series number drawn corresponds to a return with a small probability of large loss or Jackpot (series 7 to 10). It is necessary to proceed as follows: let us suppose for example that candidate choose a lottery with $5 \%$ of chances to have $-20 \%$ and $95 \%$ of chances to have $+10 \%$. It is necessary that the individual draw a paper in a ballot box containing 19 papers write "max" and 1 paper written "min". If it draws the min, it receives -20\% to multiply by the basic amount and if it draws max, it receives $10 \%$ to multiply by the basic amount.

However, for series 7 to 10 , the probabilities of weak loss or jackpot vary from a lottery to another for the same individual and the yields vary from one individual to another.

We present in the tables (1) and (2), the exhaustive lists of all the probabilities concerned, those of the lotteries with weak probability of large loss and those of the lotteries with weak probability of jackpot, as well as the number of papers "min" and "max" to put in the ballot box in each case. We will sweep all the alternatives, allowing remunerating the individuals.

${ }^{6}$ this table is posted for individuals who were connectedto the centres of the experimental laboratory only (with a word of pass particluier which the experimenter, introduced at the beginning of the experiment) and which obviously answered the 10 series. 


\subsection{Descriptive statistics}

According to summarize the statistics (see table 3) of variables in the sample, we found that the sample was homogeneous in nature. Indeed, it consisted of $51.14 \%$ men. The average age was 27.341 years. Specifically, $87.33 \%$ of the population is at an age less than 25 and $10.22 \%$ of the respondents had an age between 26 and 40 years. However, the most common marital status was the single one with $73.33 \%$ while the married represented only $18.92 \%$ in the sample. Moreover, $59.26 \%$ of the respondents considered themselves as novices, $29.39 \%$ rather experienced, $10.35 \%$ and $1.00 \%$ experienced and very experienced. The primary objective of investment is for $4.90 \%$ of respondents to leave a legacy, $4.17 \%$ for training, $2.47 \%$ for saving for retirement, $11.10 \%$ for constructing a safety mattress, $17.92 \%$ to generate long-term revenue, $16.22 \%$ to generate regular income, $20.09 \%$ for buying or renovating a house, $13.20 \%$ for durable goods purchases and $1.67 \%$ to grow their wealth. Other goals are so less likely that they can be broadly represented as a secondary objective. Regarding the style of investors, $8.82 \%$ of respondents accept no fluctuations, $28.02 \%$ of respondents accept short-term fluctuations, $53.44 \%$ of respondents accept moderate fluctuations and $9.72 \%$ of respondents accept significant fluctuations. During a period of rise and after loss, $26.07 \%$ of the respondent paid immediately, $54.63 \%$ waited a turnaround and $22.39 \%$ kept rising. However, $19.65 \%$ of respondents have already invested, $21.97 \%$ of respondents intend to reallocate some of their capital share in order to achieve their goal, $58.39 \%$ of respondents intend to gradually build capital in the coming years in order to achieve their main goal, while $0.13 \%$ did not intend to invest. After a high loss, $26.07 \%$ of respondents immediately liquidate. $54.63 \%$ of respondents expect turning. Finally, $22.39 \%$ of respondents continue. However, following a decline, $26.32 \%$ of investors immediately liquidate. $43.01 \%$ of respondents liquidate after significant loss. Similarly, $30.67 \%$ of respondents remain and accept all the changes. However, $45.00 \%$ of respondents have inheritance. While $24.14 \%$ of respondents did not real estate. More than half of the sample has less than a year of investment experience. $32.97 \%$ of respondents chose a time horizon of 5 years. The average horizon reported by respondents is 10.333 years. Each respondent made three successive choices. The distribution of responses from individuals to sets of lotteries, summarized in Table (4), shows that the percentage of respondents in the category of less risk averse "RRR" is higher than the percentage of the most risk-averse "SSS". We can say that a priori, individuals are most likely to be very sunny or very careful players.

\section{ECONOMETRIC MODEL: RANDOM EFFECTS ORDERED PROBIT MODEL}

When we observe population $N$ decision makers presumably independent and identical, placed toward the same whole of choice between lotteries, emerges unobservable problems of heterogeneity of the preferences. Part of each decision-making process remains unquantifiable, and must be considered as random by the modelisator.

However, because of effect of framing or lack of concentration or others, the individual answers to a series of lotteries return sometimes to a disturbed vision of the individual aversion to the risk Manki (1977). The application of the techniques of panel to the models of discrete choice will make it possible to gum these noises. According to Palma and Picard (2002), the results obtained from students show that the variations of the risk to the risk for the same individual reacting to various risky situations are of the same order of magnitude as the variations of the average aversion to the risk individual of the various respondents.

As the aversion to the risk is a qualitative variable ordered, we consider the random effects ordered probit model, where there is an additional error normally distributed through the individuals. We propose to the guarantors 10 series of 3 lotteries, and we use techniques of panel to estimate the way in which the aversion with the risk varies at the same time between individuals and, for the same individual, of a series of lotteries to the other.

\subsection{Definition of the model}

The random effects ordered probit model, is written:

$$
y_{i k}^{*}=x_{i k} \beta+\varepsilon_{i k}, \quad \forall i=1, \cdots, N, \quad k=1, \cdots, K
$$

$y_{i k}^{*}$ : the latent variable non observed interpreted like the risk aversion in the investment. The analyst observes, $y_{i k}$ who takes the values $\{0,1, \cdots, m\}$ according to the position relating of $y_{i k}^{*}$ relative to a whole of ordered thresholds:

$$
y_{i k}=\left\{\begin{array}{l}
0, \text { if } \mathrm{y}_{i k}^{*} \leq 0 \\
1, \text { if } v_{0}<y_{i k}^{*} \leq v_{1} \\
2, \text { if } v_{1}<y_{i k}^{*} \leq v_{2} \\
\cdots \\
m, \text { if } v_{m-1} \leq y_{i k}^{*} \cdot(2)
\end{array}\right.
$$

where, $y_{i k}$ measure the risk aversion (standardized, ordinal) of individual $i$ when it answers the series $k$, 
$i$ : represent the individual investor $i=1, \cdots, N$

$k$ : represent the series of lotteries filled by the investor $k=1, \cdots, K$.

$x_{i k}:$ is the set of the whole explanatory variables specific to the individual $i$ and the series of lottery $k$ (these variables depend not only on individual $i$ and its project of investment, but also of the series $k$ of lotteries concerned). $\beta$ : Vectors of the parameters to be estimated.

\subsection{Assumptions of the model}

The random term $\varepsilon_{i k}$ is written as sum of individual term $u_{i}$ and a residual term $v_{i k}$ according to a centered normal law reduced and varying independently from one series to another for the same individual:

$$
\varepsilon_{i k}=v_{i k}+u_{i}
$$

where $u_{i}$ :term corresponding to heterogeneity non observed for the individual $i$, presumably random and independently distributed $x_{i k}$. We suppose that $u_{i}$ follows $N\left(0, \sigma_{u}^{2}\right)$, where $\sigma_{u}^{2}$ is to be estimated $v_{i k}$ : Normally distributed random term, corresponding to the errors of measurement specific to the series of lotteries.

Following the problem of identification, we suppose $\sigma_{v}^{2}=1$, from where $v_{i k}$ follows $N(0>, 1)$.

We preserve under a condition of orthogonally of the disturbances with the random parameters of taste, simple formulations of the probabilities of ordered probitmoddel obtained under assumption of independence and identicity of the disturbances, while generating the existence of unobservable correlations between the various options of choice:

$$
\begin{gathered}
E\left(v_{i k} \backslash X\right)=0, E\left(u_{i} \backslash X\right)=0, \operatorname{cov}\left(v_{i k}, u_{j} \backslash X\right)=0, \forall i, k, j \\
\operatorname{cov}\left(v_{i k}, v_{j s}\right)=\left\{\begin{array}{c}
\operatorname{var}\left(v_{i k}\right)=1, i f \mathrm{i}=\mathrm{j} \text { et } \mathrm{k}=\mathrm{s} \\
0, \text { otherwise. }
\end{array}\right. \\
\operatorname{cov}\left(u_{i}, u_{j}\right)=\left\{\begin{array}{c}
\operatorname{var}\left(u_{i}\right)=\sigma_{u}^{2}, \text { ifi }=\mathrm{j} \\
0, \text { otherwise. }
\end{array}\right.
\end{gathered}
$$

where $X$ : represent all the exogenic data of the sample, $x_{i k}, \forall i a n d k$

Consequently, the within-individual variance of the non-explained component is standardized with one, whereas the between-individual variance of the not explained component is noted $\sigma_{u}^{2}$. Therefore, the term of random error total $\varepsilon_{i k}$ is:

$$
E\left(\varepsilon_{i k} \backslash X\right)=0, \operatorname{var}\left(\varepsilon_{i k}\right)=\sigma_{v}^{2}+\sigma_{u}^{2}=1+\sigma_{u}^{2}, \operatorname{corr}\left(\varepsilon_{i k}, \varepsilon_{i s}\right)=\rho=\frac{\sigma_{u}^{2}}{1+\sigma_{u}^{2}}
$$

Although the errors $u_{i}$ and $v_{i k}$ are supposed to be independent between them and of the explanatory variables $x_{i k}$, the term of random error total $\varepsilon_{i k}$ of the various series $k$ for the same individual $i$ is correlated due at thecommon run $u_{i}$. However, this modeling is slightly different from that generally used. Indeed, $v_{1}$ is standardized to zero and the constant is included in the list of the redressers. Here, the constant is equal to zero and estimate $v_{1}{ }^{7}$.

$$
\begin{gathered}
P\left(y_{i k}=0 \backslash x_{i k}, u_{i}\right)=P\left(y_{i k}^{*} \leq v_{1} \backslash x_{i k}, u_{i}\right)=\Phi\left(v_{1}-x_{i k} \beta-u_{i}\right) \\
P\left(y_{i k}=1 \backslash x_{i k}, u_{i}\right)=P\left(v_{1}<y_{i k}^{*} \leq v_{2} \backslash x_{i k}, u_{i}\right)
\end{gathered}
$$

\footnotetext{
${ }^{7}$ this choice is arbitrary and equivalent to the paremtrisation of Green(2002)
} 


$$
\begin{gathered}
=\Phi\left(v_{2}-x_{i k} \beta-u_{i}\right)-\Phi\left(v_{1}-x_{i k} \beta-u_{i}\right) \\
P\left(y_{i k}=m \backslash x_{i k}, u_{i}\right)=P\left(y_{i k}^{*} \geq v_{m} \backslash x_{i k}, u_{i}\right)=1-\Phi\left(v_{m}-x_{i k} \beta-u_{i}\right)
\end{gathered}
$$

\subsection{Properties and identification of the model}

The function of log-likelihood is written:

$$
L=\sum_{i=1}^{n} \ln \left(P\left(y_{i 1}, y_{i 2}, \cdots, y_{i K}\right)\right)
$$

where while simply generalizing the argument of Butler and Moffitt (1982),

$$
\begin{gathered}
P\left(y_{i 1}, y_{i 2}, \cdots, y_{i K}\right)=\int_{a_{i 1}}^{b_{i 1}} \cdots \int_{a_{i K}}^{b_{i K}} \varphi\left(\varepsilon_{i 1}, \cdots, \varepsilon_{i T}\right) d \varepsilon_{i K} \cdots d \varepsilon_{i 1} \\
=\int_{-\infty}^{\infty} \Pi_{k=1}^{K}\left[\Phi\left(b_{i k} \backslash u_{i}\right)-\Phi\left(a_{i k} \backslash u_{i}\right)\right] d u_{i} \\
=\int_{-\infty}^{+\infty}\left(\Pi_{i=1}^{N}\left[\Phi\left(b_{k i}-u_{i}\right)-\Phi\left(a_{k i}-u i\right)\right]\right) \varphi\left(u_{i}\right) d u_{i}
\end{gathered}
$$

\section{INDIVIDUALS HETEROGENEITY AND RANDOM EFFECTS}

\subsection{Results}

The random effect ordered probit model is estimated by the log-likelihood function introduced by Butler and Moffitt (1982) and the method of squaring of Gauss-Hermit in agreement with the structure of the model for random effects. The version of Stata 8.2provides the order "reoprob", developed by Frechette (2001) to estimate the random effects ordered probit model, summarized in table (5).

To test the random effects, we examine the statistical significanceof $\rho$, by using the statistical test of Wald $\left(w=\frac{\rho^{2}}{S_{\rho}^{2}}\right)$. If $w>\chi^{2}$ critics value (3.84 for a criticizes level of $95 \%$ ), we reject the nullity of rho $=0$ (Green, 2002). Like, $\operatorname{corr}\left(\varepsilon_{i k}, \varepsilon_{i s}\right)=\rho=\frac{\sigma_{u}^{2}}{1+\sigma_{u}^{2}}$, if the random effect exists, then $\varepsilon_{i k}$ and $\varepsilon_{i s}$ is correlated for the same individual $i$, but not correlated through the individuals. However, if the random effects are not significant, $\sigma_{u}^{2}=0$ and $\rho=\frac{\sigma_{u}^{2}}{\left(1+\sigma_{u}^{2}\right)}=0$, which indicates that there is no correlation between the series of lotteries according to $\varepsilon_{i k}$, for the same individual.

During the estimate of the random effects ordered probit model in the tunisian sample (see table 5), we found a $\rho$ with a significant coefficient with the threshold of $1 \%$. Therefore, there is well heterogeneity between the individuals of the Tunisiansample. Moreover we carried out a test of Wald with $w=\frac{\rho^{2}}{\sigma_{\rho}^{2}}>3.84$ for a criticize level of 95\%, we reject the nullity of $\rho=0$ (Green, 2002). Consequently, there is a significant effect of heterogeneity between the individuals of the Tunisian sample. Therefore, there is a correlation between the series of lotteries for the same individual, but not through the individuals of the Tunisian sample.

In the table of estimate of a random effects ordered probit model (table 5), only the sign of the variables indicates in which direction the probability changes.

- $\quad$ Results presented show that the explanatory variable "woman" is non-significant in the Tunisian sample, with the conventional threshold of $10 \%$. This result not converging with certain results of the existing literature (see Jorg, 2003) but converges with others (see Brachinberg et al., 1999; Chattopadhyay and Duflo; 2004). Thus, the 
kind is not a factor determining of the risk aversion in the context of the financial investments in the Tunisian sample.

In addition, several variables influence with the rise the probability that the aversion with the risk increases. Precisely:

- $\quad$ dummy relating to the age of the individual, only the variable "age2" corresponding to the investors whose age is between 26 and 40 years, influences with the rise the probability that the risk aversion increases, with a coefficient positive andsignificant with the threshold of $1 \%$ compared to the category of reference "age 1 " corresponding to the investors whose age is less than 25 years. However, the variables "age3" and "age4" are nonsignificant with the conventional threshold of $10 \%$.

- $\quad$ in the same way, all explanatory variables representing the style of the investor "investstyle2" which do not accept any fluctuation and "investstyle3" relating to that which accepts that fluctuations of short durations are favorable also to theprobability of increase in the risk aversion with coefficients positive and significant with the common threshold of $1 \%$ compared to the category of reference "investstyle5" corresponding to the investor who accepts even the significant fluctuations, while the other variables in the model are supposed to be constant. However, the dummy "investstyle4" representing the investors who accept that moderate fluctuations is non-significant with the conventional threshold of $10 \%$.

- $\quad$ variables representing types of lotteries in the questionnaire, are such as the dummy "lottype2", "lottype3" and "lottype4" corresponds respectively to the law of the uniform, binary lottery equiprobable and a small probability of great profit, is an increasing function of the risk aversion and with positive and significant coefficients with the common threshold of $1 \%$ compared to the category of reference "lottype5" corresponding to the lottery of weak probability of Jackpot, while the other variables in the model are supposed to be constant.

- In the same way, the variable representing the type of transformation of the lotteries individual faces at the time of his choice is: "Addmult1" transformations additive, is significantly different compared to the category of reference "addmult2" indicating that the transformation of the lottery is multiplicative, with a positive and significant coefficient with the threshold of $5 \%$. This results in the fact that the additive transformations of the lotteries influence with the rise the probability that the aversion increases, while the other variables in the model are supposed to be constant.

- $\quad$ Variable "lambdaAdd" representing the additive scale effects is significant with the threshold of $5 \%$, with a positive coefficient. This result indicates that the "lambdaAdd" influence with the falls the probability that the risk aversion increases, while the other variables in the model are supposed to be constant. Moreover, the variable "lamdaMult" is significant with also positive coefficients with the threshold of $1 \%$.

On the other hand, the assumption of convergence is also supported by negative coefficients of the following variables:

- Objective of the investment is important to determine the individual risk aversion. Indeed, for "obj6" representing the objective to build a mattress of safety. The other objectives are non-significant with the conventional threshold of $10 \%$.

- In addition, an original result ${ }^{8}$ is obtained. It consists so that the married respondents are less risk averse. Indeed, the dummy "matri1" relating to the married individuals is significantly different from the category of reference "matri7" that of the single people, with negative coefficient and significant with the threshold of $10 \%$. However, the variables "matri5", "matri6" and "matri8" respectively representing the individuals divorced, widowed and in couples but nonmarried are non-significant with the conventional thresholds of $10 \%$.

Lastly, the variable "Dummyinvestcapital" representing individuals having chosen capital to less invest an amount than 50000 Euros, the variables representing the behavior of the investor after a loss, "lossbehave2" indicate that the investor liquidates immediately after a loss, "lossbehave3" corresponding to the investor which liquidates only after significant loss, "losswinsbehave2" corresponding to the investor which liquidates immediately and "losswinsbehave3" corresponding to the investor who awaits reversal after a loss, are no significant with the conventional threshold of $10 \%$, when the other variables in the model are supposed to be constant.

Finally, we note that all the thresholds are positive and significant with the thresholds of $1 \%$, except for the first threshold.

\subsection{Discussion of the random effects}

After testing the heterogeneity of the individuals, moved by the will to study the impact of the latter on our model, we ask ourselves the following questions: does the random effects always improve the explanatory power of the model? In addition, how is the significance of the explanatory variables affected by unobservable heterogeneity of the individuals? To answer these questions, we will compare the level of significance of the variables and their signs, in the two estimates with and withoutrandom effects. We compare the tables of the results of estimate of the random effects ordered probit model presented in the table (5) and that of ordered probit model presented in the table $(6)^{9}$. We note that the model probit ordered with random effects in the Tunisian sample, improves the statistics of Log likelihood. Indeed, the results of statistics of the probability of notation are (LL: Log likelihood $=-7045.259$ ) in random effects ordered probit model, whereas it was smaller (Log likelihood $=-7233.246)$ in the ordered probit model. Therefore, the Tunisian sample indicates that the taking into account of the heterogeneity of the individuals in general improves the explanatory power of the models. However, in the Tunisian sample we did not find improvement of pseudo- $R^{2}$ in the random effects ordered probit model (pseudo- ${ }^{2}$ $=1.89 \%$ ) compared to the ordered probit model(pseudo- $\mathrm{R}^{2}=2.81$

${ }^{8}$ this result is also found at the time of the regression probit ordered ${ }^{9}$ Pour more detail sweats the ordered probit model to see appendix B 
In addition, we will see how the significance of the explanatory variables is affected by the unobservable heterogeneity of the individuals. Thus, we compare the results of the regressions in the sample, of the ordered probit model and random effects ordered probit model, we note that the random effects ordered probit model weakens the significance of many explanatory variables. On the other hand, the signs of all the explanatory variables remain unchanged. Precisely, in the regression with random effects, we have completly lost the significance with the conventional threshold of $10 \%$ of the explanatory variables "age3", "matri5", "ecoclim1", "ecoclim2", "ecoclim3", "ecoclim4", "invbehave1" and "investstyle4", respectively corresponding to the individuals whose age is between 40 and 55 years, the divorced individuals, with the individuals who think that the economic climate in three years will be definitely better or a little better or about the same one, or less good, with the individuals who think that the council of a specialist in investment significant whole and with the individuals who accept the moderate fluctuations. However, the level significance of the variables "matri1" and "obj9" dropped, while passing respectively from $1 \%$ to $10 \%$, and $1 \%$ to $5 \%$. The only variables whose level of significance improved at the time of the regression for random purposes are "Addmult1" and "lambdaMult" corresponding respectively to the transformations of additive lotteries and the scale effects multiplicative, whose thresholds passed respectively from $10 \%$ to $5 \%$ and $5 \%$ to $1 \%$. Lastly, the level of significance of the remainder of the variables did not change.

\section{VARIANCE ANALYSIS OF THE RANDOM EFFECTS}

In order to understand the factors influencing on the risk aversion of the individuals, we carry out a variance analysis on the random effects ordered probit model. This section treat the second objective of our article: we try to determine if the inequality in risk aversion in the context of the financial investments is following differences will within or between individual. Our analysis consists of a decomposition of the variance of the variable dependent on individual risk aversion, in within individual and between-individual components of the observable and unobservable components.

Precisely, by applying a discrete choice model, namely random effects orderedprobit model, we present a method to consider the within individual and the between-individual components of the variance explained and not explained individual risk aversion in the context of financial investment. This decomposition aims to study the stability of the individuals risk aversion of a series with another, by analyzing within-individual variability. We call:

Component of the within-individual variance: the within-individual contribution of the latent variable of risk aversion by those of the explanatory variables, and

Component of the between-individual variance: the between-individual contribution of the latent variable of risk aversion explained by that of the explanatory variables.

\subsection{Results of the variance analysis}

We study if the inequality in aversion with risk in the context of financial investments is following differences within or between individual. By estimating a random effects orderedprobit model, we apply a simple method to estimate the within andthe between individuals components of the explained and not explained risk aversion variance. For our empirical analysis, we use the base of the data collected at the time of the experimental laboratory led in Tunisia during the time between February and April 2012.

We measure the inequality in risk aversion of the investors by making a variance analysis, according to following stages':

- $\quad$ we start by calculating the value considered linear of the latent variable for each observation of the

Tunisian sample

$\hat{Y}_{i k T N}^{*}=\hat{\beta}^{\prime} X_{i k}$

\begin{tabular}{|c|c|c|c|c|c|}
\hline Variable & $\begin{array}{c}\text { Nbr } \\
\text { d'obs }\end{array}$ & Mean & Stand-Deviation & Min & Max \\
\hline linearPanelTUN & 3833 & 0.983 & 0.431 & -0.209 & 2.596 \\
\hline
\end{tabular}

Statistical summary of the variable linearPanelTUN

1. Then, we calculate the average of its estimated values $\hat{Y}_{i T N}^{*}$ for each individual $i$ and we generate a new variable $\hat{Y}_{i k T N}^{d}=\hat{Y}_{i k T N}^{*}-\hat{Y}_{i T N}^{*}$. The new variable $\hat{Y}_{i k T N}^{d}$ is thus a measurement of heterogeneity within individual for an individual $i$ in the Tunisian sample.

\begin{tabular}{|c|c|c|c|c|c|}
\hline Variable & Nbrobs & Mean & Stand-Deviation & Min & Max \\
\hline YikdTN & 3833 & $4.47 e-11$ & 0.235 & -0.443 & 0.605 \\
\hline
\end{tabular}


Statistical summary of the variable YikdTN

\begin{tabular}{|c|c|c|c|c|c|}
\hline Variable & Nbrobs & Mean & Stand-Deviation & Min & Max \\
\hline YiTN & 3965 & 0.983 & 0.361 & 0.035 & 2.102 \\
\hline
\end{tabular}

Statistical summary of the variable YiTN

A last stage consists in calculating, the variances of $\hat{Y}_{i T N}^{*}$ et $\hat{Y}_{i k T N}^{d}$ :

$V\left(\hat{Y}_{i T N}^{*}\right)=0.131$ is the explained between-individual variance,

$V\left(\hat{Y}_{i k T N}^{d}\right)=0.055$ is the explained within-individual variance,

$V\left(u_{i}\right)=\sigma_{u}^{2}=0.369^{10}$ is the non-explained variance of the between-individual component,

$V\left(v_{i k}\right)=1$ is the non-explained variance of the within-individual component.

The total variance is $: V\left(\hat{Y}_{i k T N}^{*}\right)=V\left(\hat{Y}_{i T N}^{*}\right)+V\left(\hat{Y}_{i k T N}^{d}\right)+V\left(v_{i k}\right)+V\left(u_{i}\right)=1.556$

\subsection{Interpretation}

We note according to the table (5) which the fraction of the explained variance is much weaker than that not explained $(11.98 \%$ against $87.99 \%)$ in the random effects orderedprobit formulation in the sample. This suggests that variation of the risk aversion of individuals is probably not explained by the observable characteristics. Therefore, in the variance analysis with the random effects orderedprobit model, we explain only $11.98 \%$ of the original variance of the sample, which is a rather good result.

In addition, the majority of the explained variance must with the between-individual component with $8.42 \%$. Indeed, the weight of the explained within-individual component is 3.56, whereas the percentage of the between-individual explained variance is $8.42 \%$. Concerning the not explained part of the original variance, we find that the within-individual variance is $64.28 \%$ much higher, with the between-individual variance of $23.73 \%$. These results show that the inequalities of the risk aversion between individual (between-individual exp + between individual not $\exp =8.42 \%+23.73 \%=32.15 \%$ ) are less significant than those within-individual individuals (within-individuals.

exp+within-individuals.nonexp=3.56\%+64.28\%=67.84\%).

In conclusion, we explained approximately $12 \%$ of the original variance and we found that it mainly had within and between-individual difference. However, overall the inequality in the risk aversion must mainly be concerned with the difference within individual. From where, the non-stability of the answers of the individuals of a series of lottery to another. However, like the within-individual variance, is that relating to the stability of the answers of the individuals of a series of lottery to another, we can say that a significant component of the variability of the risk aversion must go with the variability of the answers of the individuals through the series of lotteries. However, component within individual not explained is more significant than that explained in the sample. Consequently, there is no stability of the answers of the individuals of one series to the other.

\section{CONCLUSION}

The study of risk aversion in the behaviors of financial investments occupies a dominating place in the theoretical and empirical recent literature. In our work, we tried to quantify its importance and to determine its constitutive factors. Indeed, ifthe analysis of the financial products were largely treated, the analysis of the attitude of the investor in particular his psychological aptitude to take risks, remains little exploited. In order to give an account of certain deviations compared to the theory of expected utility, we introduced individual heterogeneity. Indeed, the same individual confronted with identical conditions answers differently, the same questions during different occasions. The classical theory of expected utility is not able togive an account of this simple observation. We can argue that these variations of behaviors can be considered as errors, present in any data acquisition. This taking into account of the errors, from the start at the theoretical level, seemed significant. The importance of this extension lies in the fact that it applies as well to the model of expected utility as with the non-expected utility model. With an aim of controlling this individual heterogeneity, we used for our estimates the random effects ordered probit model. Indeed, there is an unobserved heterogeneity of the population tastes: the parameters of the variables are not invariants with the individual sampled.

Initially, we tested and controlled the heterogeneity of the individuals in the sample. We showed that there is heterogeneity

${ }^{10}$ According to the table (5), $\rho=\frac{\sigma_{u}^{2}}{1+\sigma_{u}^{2}}=0.270$, then $\sigma_{u}^{2}=\frac{0.270}{1-0.270}=0.3698$. 
between the individuals well. In addition, a narrow examination of the estimates through these models proved that, contrary to the shared results, the control of the random effects in general has little effect on the estimates and their significance. Indeed, by comparing this model, the risk aversion correctly estimated proved that the models consideringenvisage better the choice of the investor at the time of the regression ordered probit model that in that for random effects. However, log-likelihood is improved during the estimate for random effects.

In the second time, we tried to measure the inequality of the risk aversion of the individuals, by carrying out a variance analysis on the random effects orderedprobit model. We presented a method allowing measuring the components of between and within individual of the risk aversion. Indeed, understand the relative importance of the differences well within and between individual of the risk aversion is crucial, be given their impact on the choices of the individual. However, we know little on the magnitudes relating to the inequalities in the within and between individual of the risk aversion. According to the variance analysis, we showed that the differences between the variances between and within individual are significant. In addition, this, since we found that the inequality in the risk aversion must mainly with the difference within individual.

According to the variance analysis, we found that our model explains $12 \%$ of original variance of the risk aversion. The differences between the variances between and within individual are significant. Since we found that, the inequality in the risk aversion must mainly with the difference within individual. However, the within-individual components are more significant in the sample for the variance observed and less significant in the sample for the variance not observed. We can say that the within-individual variance must mainly with the not explained component. In addition, the share of the variance of the risk aversion had with the component of the between-individual variance in the sample is $32.14 \%$.

Overall, we showed that the inequality in risk aversion in the within-individual component were much more significant than those between individual. However, like the within-individual component variance, is that relating to the stability of the answers of the individuals of a series of lottery to another, we could affirm that a significant component of the variability of the risk aversion must with the variability of the answers of the individuals through the series of lotteries. However, it should be noted that the non-explained within-individual component is more significant than that explained. While, the explained component of the variance, must mainly with the between-individual variance. In addition, the techniques of the discrete choice models make it possible to test certain refinements of the expected utility theory. And this, by putting the same individuals in the similar from the point of view of theory of traditional expected utility, but appreciably different situations. Thus, according to the ideas of Kahneman and Tversky (1979), we find indications of non-derivability of the utility function around zero. Therefore, individuals are more risk averse in the losses. This deviation will be the subject of an extension of this article.

\section{REFERENCES}

[1] Anderson, S., A. de Palma and J.-F. Thisse (1992), Analysis of Differentiated Markets with Discrete Choice Models, MIT Press, $423 \mathrm{p}$.

[2] Barsky R.B., Kimball M.S., Juster F.T. et Shapiro M.D. (1997), Preference Parameters and BihavioralHeterogeneity:An experimental approch in the health and retirement survey, Quarterly Journal of Economics, vol.112, n.2, pp. 537-580.

[3] Ben-Akiva, McFadden, Train, Walker,Bhat, Bierlaire, Bolduc (2001), Hybrid Choice Models : Progress and Challenges, Marketing Letters vol.13, n. 3, pp. 163-175.

[4] Ben-Akiva M., McFadden D., Garling T., Gopinath D., Walker J., Bolduc D. (1999), Extended Framework for Modeling Choice Behavior, Marketing Letters, vol. 10,no. 3.

[5] Brachinberg H. W., Brown M., Gysler M., Schubert R. (1999), Financial decision-making : Are women really more risk-averse?, The American Economic Review, vol. 89, pp. 381-385.

[6] Broihanne, M.-H., Merli M., Roger P. (2004), Finance Comportementale, Economica.

[7] Brooks P., Zank H. (2004), Attitudes on Gain and Loss Lotteries: A Simple Experiment, School of Economic Studies, The University of Manchester, United Kingdom.

[8] Butler LS., Moffitt R. (1982), A computationally efficient quadrature procedure for the one factor multinomial probit model. Econometrica vol. 50, pp.761-764

[9] Chamberlain G. (1984), Panel Data, in Z.Griliches and M.Intriligatoreds, Handbook of Econometrics, vol.2, pp.1247-1318.

[10] Chattopadhyay R., Duflo E.(2004), Women as Policy makers: evidence from a randomised policy experiment in India, Econometrica, vol. 72, n.5, pp.1409-1443.

[11] de Palma, A. et N. Picard, (2002), Measuring Risk tolerance on the web, Manuscript. University of Cergy-Pontoise, THEMA, France.

[12] de Palma A., Picard N.(2005), Route choice decision under travel time uncertainty, Transportation Research Part A, vol. 39, n.4, pp.295-324.

[13] de Palma, A., Ben-Akiva, M., Brownstone, D., Holt, C., Magnac, T., McFadden, D., Moffatt, P., Picard, N., Train, K., Wakker, P., Walker, J. (2008), Risk, uncertainty and discrete choice models, Marketing Letters, vol. 19, pp. 269-285.

[14] Donkers B., Melenberg B. et van Soest A. (2000), Estimating risk attitudes using lotteries; a large sample approach. Tilburg University, Department of Econometrics and CentER, The Netherlands.

[15] Eeckhoudt L., Gollier C., Schlesinger H., (2005), Economic and Financial Decisions Under Risk, Princeton University Press.

[16] Frechette GR.(2001), Random effects ordered Probit, Stata Tech Bull vol. 159, pp. 23-27.

[17] Goeree, J., C. A. Holt, Palfrey T. (2003), Risk Averse Behavior in Generalized Matching Pennies Games, Games and Economic Behavior, vol. 45, pp. 97-113.

[18] Gollier, C (2001), The Economics of Risk and Time, The MIT Press, Cambridge, MA. 
[19] Green, William H. (2002), Econometric Analysis, 6nd ed, Englewood Cliffs, NJ: Prentice Hall.

[20] Hartog J., Ferrer-i-Carbonell A. et Jonker N. (2000), On a simple survey measure of individual risk aversion, CESifoWorking Paper Series, n.363.

[21] Hedeker D., Gibbons R. (1994), A random-effects ordinal regression model for multilevel analysis. Biometrics, vol. 50, pp. 933-944.

[22] Holt C. A., Laury S. K. (2003), Risk Aversion and Incentive Effects, American Economic Review, vol. 92, pp. 1644-1655.

[23] Holt C.A.(2006), Markets, Games, and Strategic Behavior, Boston: Addison-Wesley.

[24] Kahneman D., Tversky A. (1979), Prospect theory: An analysis of decision under risk, Econometrica, vol.47, n.2, pp.263-291.

[25] Luce D. R. (2000), Utility of Gains and Losses: Measurement-Theoretical and Experimental Approaches, Lawrence Erlbaum Publishers, London.

[26] McFadden D.(2001), Economic Choices, American Economics Review, vol.91, n. 3, pp.351-378.

[27] Roth A. (1988), Laboratory Experimentation in Economics: A Methodological Overview, The Economic Journal, vol. 98, pp. 974-1031.

[28] Train K., McFadden D. (2000), Mixed MNL models for discrete response', Journal of Applied Econometrics, vol.15, pp.447-470.

[29] Tversky, A., Kahneman, D. (1992), Advances in prospect theory : cumulative representation of uncertainty, Journal of Risk and Uncertainty, vol. 5, pp. 297-323.

[30] Von Neumann J., Morgenstern O. (1947), Theory of Games and Economic Behaviour, Princeton University Press, Princeton.

[31] Walker, Ben-Akiva (2002), Generalized Random Utility Model', Mathematical Social Sciences, vol. 43, n.3, pp. 303-343.

\section{Appendices A}

\begin{tabular}{|c|c|c|c|}
\hline $\begin{array}{c}\text { Series 7 and 8 "weak } \\
\text { probability of large loss" }\end{array}$ & $\begin{array}{c}\text { Probability of the } \\
\text { mnimum }\end{array}$ & Numbers "min" & Nombre "max" \\
& & & \\
\hline & 0.5 & 1 & 200 \\
\hline & 1 & 1 & 99 \\
\hline & 3 & 3 & 97 \\
\hline & 6 & 6 & 94 \\
\hline & 9 & 9 & 87 \\
\hline & 13 & 13 & 83 \\
\hline
\end{tabular}

Table 1. Table of renumeration of the lotteries with weak probability of large loss

\begin{tabular}{|l|c|c|c|}
\hline $\begin{array}{l}\text { Series 9 and 10 "weak } \\
\text { probability of Jackpot" }\end{array}$ & $\begin{array}{c}\text { Probability of the } \\
\text { mnimum }\end{array}$ & Numbers "min" & Nombre "max" \\
& & & \\
\hline & 85 & 85 & 15 \\
\hline & 88 & 88 & 12 \\
\hline & 90 & 90 & 10 \\
\hline & 92 & 92 & 8 \\
\hline & 93 & 93 & 6 \\
\hline & 94 & 94 & 5 \\
\hline
\end{tabular}

Table 2. Table of renumeration of the lotteries with weak probability of jackpot 


\begin{tabular}{|c|c|c|c|c|c|}
\hline Variable & Mean & (Std. Dev.) & Min. & Max. & $\mathbf{N}$ \\
\hline yordC & 4.101 & (2.693) & 1 & 8 & 3867 \\
\hline Indicatrice femme & 0.489 & $(0.5)$ & 0 & 1 & 4001 \\
\hline agesuperieur 25 ans et inferieur ou egal a 40 ans & 0.301 & $(0.459)$ & 0 & 1 & 4001 \\
\hline $\begin{array}{c}\text { agesuperieur a } 40 \text { ans et inferieur ou egal a } 55 \\
\text { ans }\end{array}$ & 0.061 & $(0.239)$ & 0 & 1 & 4001 \\
\hline agesuperieur a 55 ans & 0.011 & $(0.104)$ & 0 & 1 & 4001 \\
\hline matrimony $==$ Marie avec contrat & 0.189 & $(0.392)$ & 0 & 1 & 4001 \\
\hline matrimony $==$ Divorce & 0.011 & $(0.104)$ & 0 & 1 & 4001 \\
\hline matrimony $==$ Veuf & 0.002 & $(0.05)$ & 0 & 1 & 4001 \\
\hline en couple non marie & 0.064 & $(0.245)$ & 0 & 1 & 4001 \\
\hline ecoclim $==$ Nettement meilleur & 0.124 & $(0.33)$ & 0 & 1 & 3977 \\
\hline ecoclim==Un peu meilleur & 0.396 & $(0.489)$ & 0 & 1 & 3977 \\
\hline ecoclim==A peu pres le meme & 0.284 & $(0.451)$ & 0 & 1 & 3977 \\
\hline ecoclim==Moins bon & 0.178 & $(0.383)$ & 0 & 1 & 3977 \\
\hline investorbehave $==P$ as du tout important & 0.017 & $(0.128)$ & 0 & 1 & 3989 \\
\hline investorbehave $==$ Un peu important & 0.163 & $(0.37)$ & 0 & 1 & 3989 \\
\hline investorbehave $==$ Important & 0.449 & $(0.498)$ & 0 & 1 & 3989 \\
\hline investstyle==Aucune & 0.088 & $(0.284)$ & 0 & 1 & 4001 \\
\hline investstyle $==$ Courte Duree & 0.28 & $(0.449)$ & 0 & 1 & 4001 \\
\hline investstyle==Moderee & 0.534 & $(0.499)$ & 0 & 1 & 4001 \\
\hline addmult $==\mathrm{A}$ & 0.596 & $(0.491)$ & 0 & 1 & 4001 \\
\hline lambdaAdd & 0.062 & $(0.074)$ & 0 & 0.31 & 4001 \\
\hline lambdaMult & 0.044 & $(0.068)$ & 0 & 0.26 & 4001 \\
\hline lottype $==1.0000$ & 0.324 & $(0.468)$ & 0 & 1 & 4001 \\
\hline lottype==2.0000 & 0.309 & $(0.462)$ & 0 & 1 & 4001 \\
\hline lottype $==3.0000$ & 0.184 & $(0.387)$ & 0 & 1 & 4001 \\
\hline maintarget==Securite & 0.111 & $(0.314)$ & 0 & 1 & 4001 \\
\hline maintarget $==$ Revenus a terme & 0.179 & $(0.384)$ & 0 & 1 & 4001 \\
\hline maintarget==Maison & 0.201 & $(0.401)$ & 0 & 1 & 4001 \\
\hline maintarget==Biens durables & 0.132 & (0.338) & 0 & 1 & 4001 \\
\hline
\end{tabular}

Table 3: Statistical summary of the Tunisian sample

\begin{tabular}{|c|c|c|}
\hline Range j & Choice & Sample \% \\
\hline
\end{tabular}




\begin{tabular}{|c|c|c|}
\hline 1 & RRR & 29.09 \\
\hline 2 & RRS & 9.80 \\
\hline 3 & RSR & 7.76 \\
\hline 4 & RSS & 9.34 \\
\hline 5 & SRR & 9.78 \\
\hline 6 & SRS & 6.90 \\
\hline 7 & SSR & 8.20 \\
\hline 8 & SSS & 19.14 \\
\hline
\end{tabular}

Table 4. Recapitulation of the choices distributions

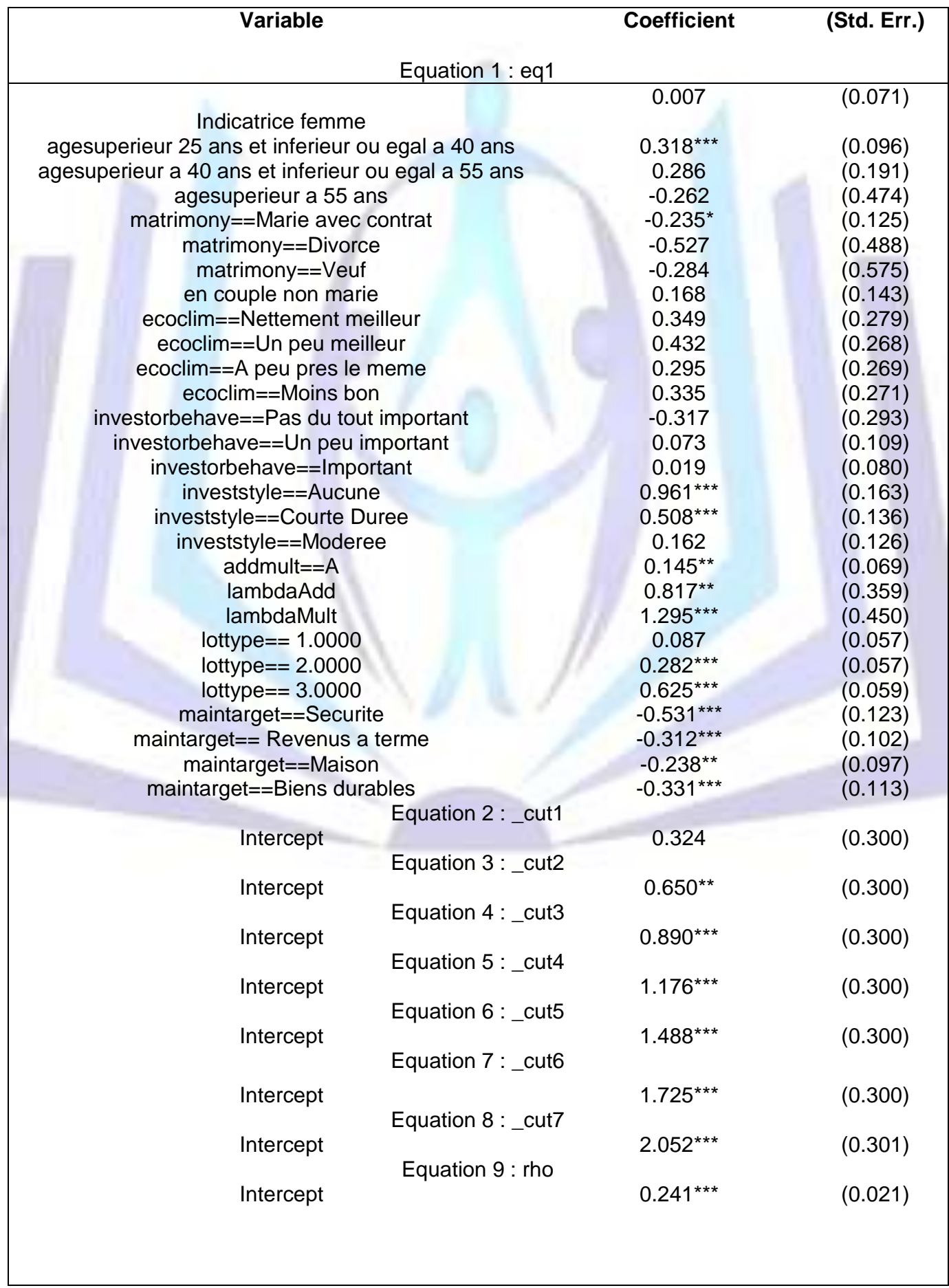




\begin{tabular}{cc|}
\hline $\begin{array}{c}\text { Number of observation } \\
\text { Number of individuals } \\
\text { Log-likelihood } \\
X^{2}(28)\end{array}$ & 3833 \\
$(383)$ \\
Variance (B : Between, W : Within, T : Total) & -7045.259 \\
B explained (\% B exp / T Total) & 271.438 \\
W explained (\% W exp / T Total) & $0.131(8.42 \%)$ \\
B non-explained (\% B non exp / T Total) & $0.055(3.56 \%)$ \\
W non-explained (\% W non exp / T Total) & $1(64.27 \%)$ \\
\hline Levels of significance : ${ }^{*}: 10 \%$ & \\
\hline
\end{tabular}

Table 5: Results of the estimate of the random effects ordered probit model

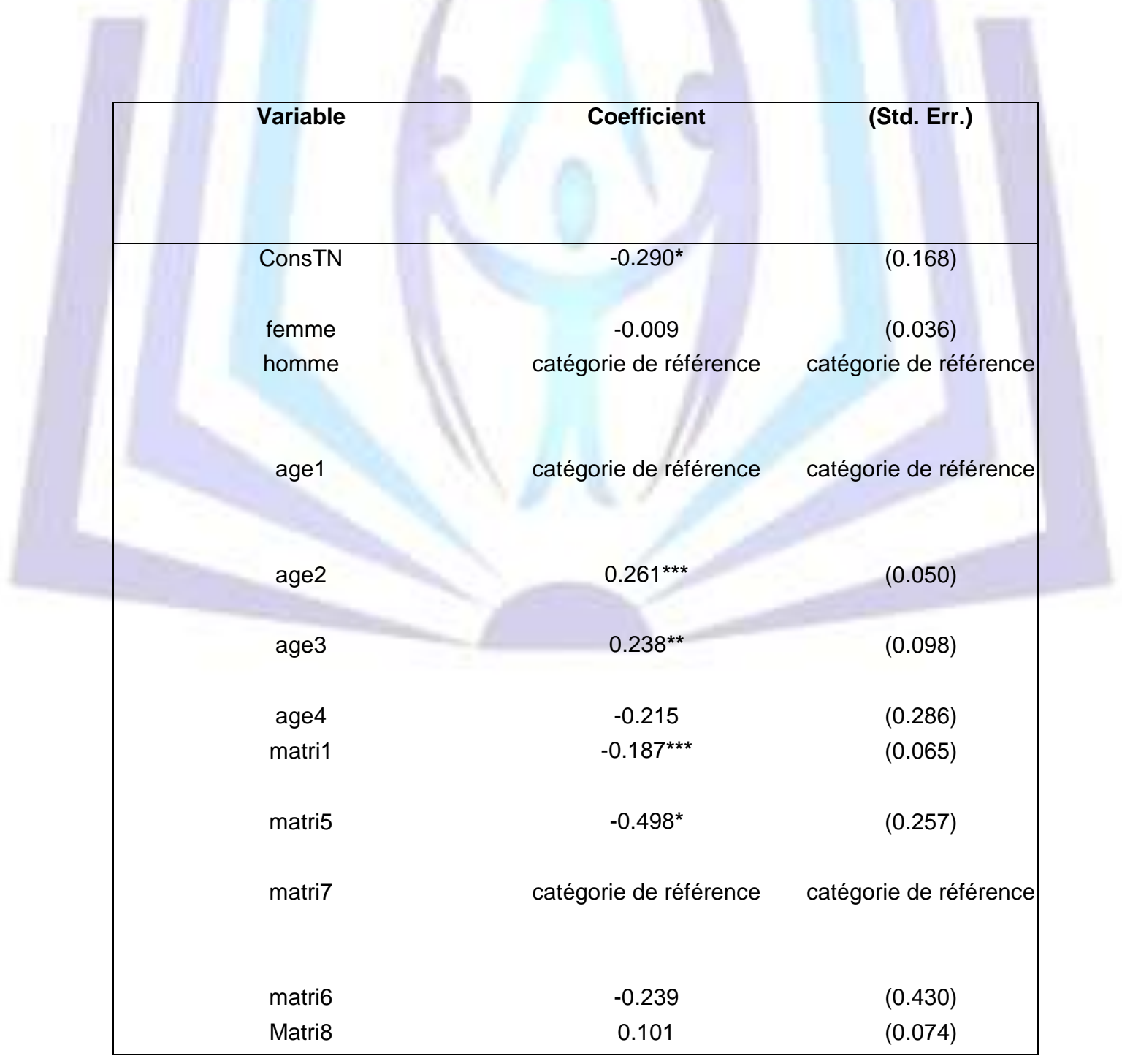




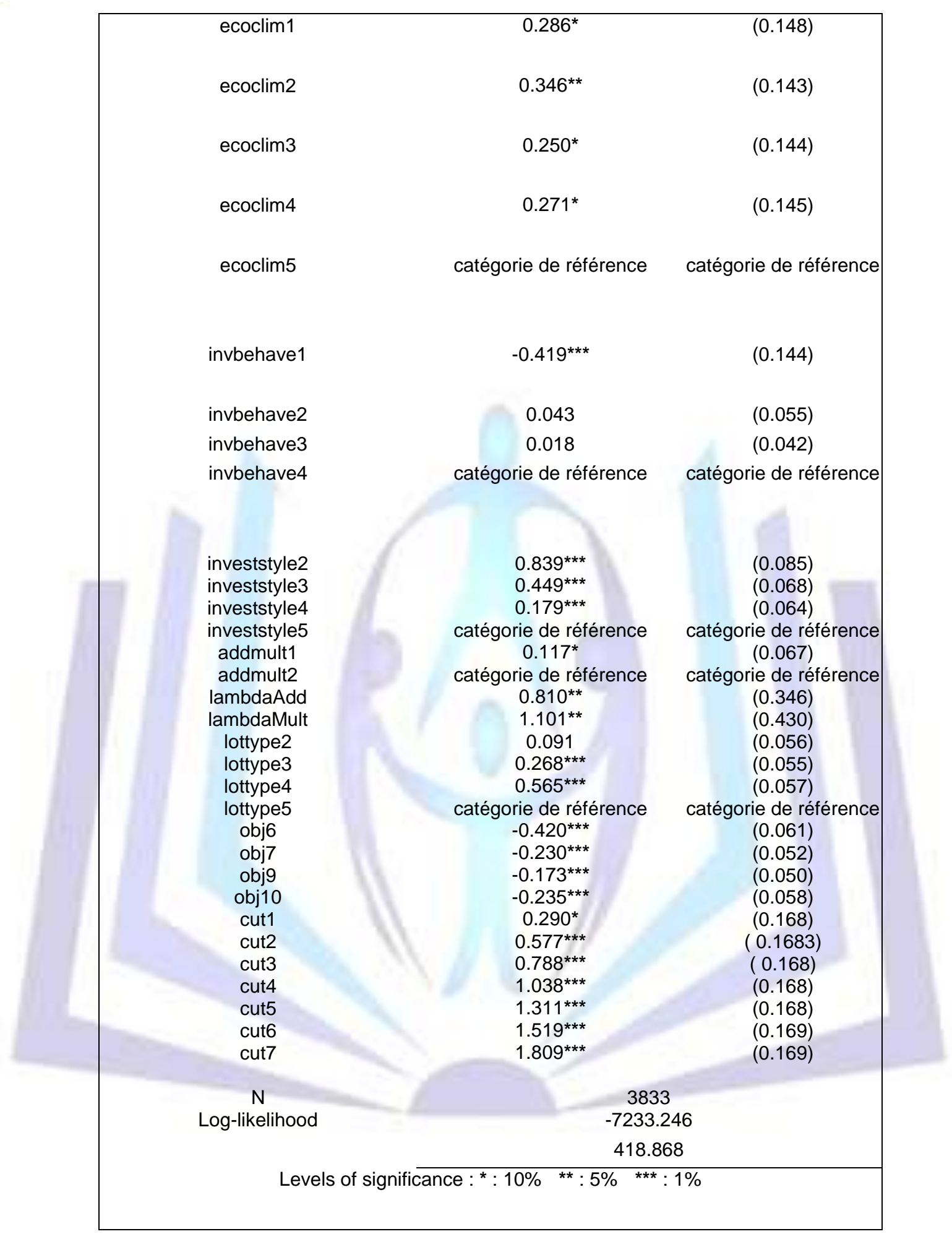

Table 6: Results of the estimate of the ordered probit model 